\section{FRI0506 CLINICAL ANALYSIS OF BEHÇET'S DISEASE MANIFESTATIONS IN 453 EGYPTIAN PATIENTS: GENDER COMPARISON}

M. Elshahaly ${ }^{1}$ I. Omar ${ }^{2}$, H. Bassiouni ${ }^{3} .{ }^{1}$ Rheumatology, physical medicine and rehabilitation, Suez canal university, Ismailia; ${ }^{2}$ Ophthalmology, Alexandria university, Alexandria; ${ }^{3}$ Rheumatology, physical medicine and rehabilitation, AlAzhar university, Cairo, Egypt

Background: Behçet's disease (BD) is characterised by the presence of vasculitis of veins and arteries of all sizes. ${ }^{1}$ Persistent oral and genital ulcers are hallmarks of the disease. BD may be associated with inflammation of eyes, joints, vessels, GIT, nervous system, and other systems. ${ }^{2} 70 \%$ of BD patients suffer from ocular complications mainly recurrent uveitis that may lead to loss of vision. ${ }^{3}$ Objectives: To describe the demographic and clinical features including ocular manifestations of $\mathrm{BD}$ in Egyptian patients and to compare the incidence of disease complications between males and females.

Methods: 453 subjects fulfilling 1990 Classification criteria for BD were included. Detailed history, physical examination and complete ocular examination were done. Both, a rheumatologist and an ophthalmologist did clinical examination and follow-up. Further comparison of disease manifestations between both genders was done. Patients were followed up every 2 weeks for 4 years for monitoring of disease complications.

Results: Median (range) age of patients was $45^{32-63}$ years old. Our sample included 297 (65.6\%) males and 156 (34.3\%) females. Patients' disease duration ranged between one to 16 years. At baseline, acute phase reactants (ESR and CRP) were significantly higher in females compared to males $(p<0.01)$ indicating a higher disease activity.

Oral ulcers were present in $100 \%$ of patients. Prevalence of genital ulcers, erythema nodosum and joint involvement was similar between both sexes. Subcutaneous thrombophlebitis and follicular papules were more common in males compared to females ( $\mathrm{p}=0.009$ and $\mathrm{p}=0.041$ respectively).

Recurrent iridocyclitis was found in $100 \%$ of patients. All ocular findings were significantly more common in females compared to males except retinal haemorrhages that were more common in males $(49.8 \%$ vs $37.2 \%)$. Incidence of secondary cataract and glaucoma in addition to vitreous opacities were similar between both sexes.

$14.8 \%$ of patients had GIT manifestations including Budd-Chiari syndrome in 10 males and 5 females; bloody diarrhoea was found in 10 males. Disturbed bowel habits were present in 27 males and 10 females.

Deep venous thrombosis (DVT) was found in $11.8 \%$ of males and $3.2 \%$ of females. Mediastinal vein syndrome was found in $3.7 \%$ of males only, pulmonary artery aneurysm in $1.3 \%$ of males and $3.2 \%$ of females. Aseptic meningitis and hemiplegia were found $3.4 \%$ and $3 \%$ of males respectively. $1.7 \%$ males and $3.2 \%$ females suffered from brain stem involvement. Superior sagittal sinus thrombosis was present in $3.4 \%$ females.

Conclusions: BD has wide range of complications most commonly oral ulcers and recurrent iridocyclitis. Surprisingly, disease activity was higher in females at baseline and ocular complications were more common in females compared to males.

\section{REFERENCES:}

[1] Nair JR, Moots RJ. Behcet's disease. Clin Med (Lond) 2017 Feb;17(1):7177.

[2] Kural-Seyahi E, Fresko I, Seyahi N, et al. The long-term mortality and morbidity of Behcet's syndrome. A 2 decade outcome survey of 387 patients followed at a dedicated center. Medicine (Baltimore) 2003:82:60-76.

[3] Kitaichi N, Miyazaki A, Iwata D, Ohno S, Stanford MR, Chams H. Ocular features of Behçet's disease: An international collaborative study. $\mathrm{Br} \mathrm{J}$ Ophthalmol 2007;91(12):1579-1582.

Disclosure of Interest: None declared

DOI: 10.1136/annrheumdis-2018-eular.2824

\section{FRI0507 THE PREVALENCE OF LATENT TUBERCULOSIS INFECTION IN INDIAN PATIENTS OF TAKAYASU ARTERITIS}

N.V. Negalur ${ }^{1}$, G. Ekbote ${ }^{1}$, D. Tanna ${ }^{1}$, N. Mendiratta ${ }^{1}$, R. Gupta ${ }^{1}$, A. Bhan ${ }^{2}$. ${ }^{1}$ Rheumatology and Clinical Immunology, ${ }^{2}$ CTVS, MEDANTA- THE MEDICITY, Gurgaon, India

Background: Studies have shown a correlation between Tuberculosis (TB) and Takayasu arteritis (TA). Some even postulate that infection with TB is required for the initiation of aortoarteritis. Hence, this project was undertaken to find an association of TB and TA by studying the prevalence of Latent Tuberculosis Infection (LTBI) in Indian TA patients.
Objectives: To study the prevalence of LTBI in Indian TA patients and see whether LTBI is more in these patients as compared to the historical cohort $(40 \%$ in Indian population).

Methods: This was a cross sectional observational study. All consecutive patients with TA (satisfying the ACR 1990 criteria) were included prospectively from a period of May 2016 to December 2017. Their clinical, laboratory and radiological data were collected after obtaining informed consent. Patients were divided into groups based on the angiographic classification. LTBI was assessed by the Mantoux test and Quantiferon TB Gold test. Mantoux test (MT) was done with 5TU and results were read after 48-72 hours. An induration more than $10 \mathrm{~mm}$ was considered positive. Quantiferon TB Gold assay (QTB) was done by ELISA technique and (patient minus control) value $>0.35 \mathrm{IU} / \mathrm{ml}$ was considered positive. A positive result of MT and /or QTB was considered positive for LTBI. Chest X-ray was included to access evidence of past or active TB lesions. Active infection was defined as clinical and microbiological and /or radiological evidence of TB. The study was approved by the Ethics committee of Medanta hospital.

Results: Out of 66 consecutive TA patients, 46 patients had tests available for LTBI and these were included in the analysis. The mean age of the cohort was 34.9 years with a median disease duration of 24 months. Males consisted of 11 patients whereas females formed the majority i.e. 35 patients (M: $F=1: 3.1$ ). Angiographic Type $\mathrm{V}(54.3 \%)$ was the commonest in the cohort followed by Type IIB (17.3\%), Type IV, I, IIA and III (15.2\%, 8.6\%, $2.1 \%$ and $2.1 \%$ respectively). LTBI positivity was present in $32.6 \%$ of the cohort; with 5 patients (10.8\%) having both tests positive. 6 patients were MT positive without being QTB positive and 4 patients were only QTB positive. Eight patients had history of Tuberculosis out of which 1 was diagnosed with TB and TA simultaneously. Four patients were diagnosed as TA during the course of Anti tubercular treatment (ATT) between 4 to 6 months, whereas the rest were diagnosed after ATT completion. The mean duration of Anti tubercular treatment was 8 months. Koch's contact was seen in 7 patients.

Abstract FRI0507 - Table 1. Patient characteristics and LTBI

\begin{tabular}{llcc}
\hline Characteristic & Value & $\begin{array}{c}\text { Percentage } \\
(\%)\end{array}$ \\
\hline $\mathrm{n}=46$ & & 13 \\
& \multicolumn{1}{c}{ MT positive only } & 6 & 8.6 \\
& QTB positive only & 4 & 10.8 \\
$\mathrm{n}=8$ & 5 & 32.6 \\
& Both MT and QTB positive & 15 & 17.3 \\
& LTBI positive & $8 / 46$ & 50 \\
& H/O TB & 4 & 12.5 \\
& TB LN Tulmonary TB & 1 & \\
& (progression) & & 25 \\
& Intestinal TB & 2 & 12.5 \\
& TBM & 1 & 12.5 \\
& Empirical ATT for PUO & 1 & 25 \\
& MT & 2 & 0 \\
& QTB & 0 & 12.5 \\
$\mathrm{n}=7$ & Both & 1 & 15.2 \\
& MT TB contact & $7 / 46$ & 14.2 \\
& QTB & 1 & 0 \\
& Both & 0 & 0 \\
\hline
\end{tabular}

Conclusions: The prevalence of LTBI in Indian Takayasu patients was $32.6 \%$ which was not higher than the population prevalence $(40 \%$ in the historical cohort). However, a larger cohort and further association studies are needed fo the relationship between TA and Tuberculosis.

Disclosure of Interest: None declared

DOI: 10.1136/annrheumdis-2018-eular.3966

\section{FRI0508 ANALYSIS OF RISK FACTORS OF ADVERSE OBSTETRICAL OUTCOME IN PATIENTS WITH TAKAYASU ARTERITIS}

N. Abisror ${ }^{1}$, A. Mekinian ${ }^{1}$, V. Le Guern ${ }^{2}$, N. Costedoat ${ }^{2}$, on behalf of Eric Hachulla, Marc Lambert, Nathalie Morel, Catherine Chapelon, Nihal Martis, Jean

Gabriel Fuzibet, Pauline Belenotti, Laure Swiader, Robin Dhote, Luc Mouthon, Francoise Sarrot-Reynault, Marc Andre, Smail Amar, Jean Baptiste Gauthier, Pascal Cathebras, A. ${ }^{1}$ Internal Medicine, Sorbonne Université, APHP, Hopital Saint Antoine; ${ }^{2}$ Internal medicine, Université Paris Descartes-Sorbonne Paris Cité, $A P H P$, Centre de référence maladies auto-immunes et systémiques rares d'lle de France, Hopital Cochin, Paris, France

Background: Takayasu arteritis (TA) is a large-vessel vasculitis that affects young women of childbearing age. Several small case-series described the pregnancy outcome in TA patients with lack of study determining risk factors associated with adverse pregnancy outcome. 
Objectives: We performed a French retrospective study, to determine the risk factors associated with obstetrical adverse outcome; and the relation between pregnancy outcome and TA disease activity.

Methods: French nationwide retrospective study of pregnancies that lasted at least 12 weeks of gestation (WG) in TA patients.

Results: Forty-three pregnancies occurred in 33 patients. The diagnosis of TA was preexisting in 29 patients, and done during pregnancy in 4.

For the 39 pregnancies in the 29 patients with a preexisting diagnosis of TA: steroids were maintained throughout pregnancy in $23 / 39$ (59\%) with a median dose of $5 \mathrm{mg} / \mathrm{day}^{2-40}$ immunosuppressive treatment during pregnancy included azathioprine $(n=9,21 \%)$ or infliximab (TNF- $\alpha$ antagonist) $(n=1,2 \%)$. For the 4 pregnancies with TA diagnosis during, only one was treated by steroids. Aspirin (100 mg/day) was used in $27 / 43$ pregnancies (63\%) and antihypertensive drugs were used in 10 pregnancies $(23 \%)$.

Before pregnancy, immunosuppressive treatment had been used in 16 patients: azathioprine $(n=10,30 \%)$, methotrexate $(n=7,21 \%)$, TNF- $\alpha$ antagonist (infliximab in 3 and adalimumab in $1 ; n=4,12 \%)$ and cyclophosphamide $(n=2,6 \%)$.

Maternal adverse events were noted in 20 pregnancies (47\%). The most frequent adverse event was arterial hypertension $(n=12 ; 28 \%)$ : 10 worsening of previous arterial hypertension and 2 de novo arterial hypertension. Other adverse events included pre-eclampsia $(n=3 ; 7 \%)$, HELLP syndrome $(n=1 ; 2 \%)$ and post-partum haemorrhage $(n=2 ; 5 \%)$. No maternal death was observed.

There were 42 live births (98\%) delivered at a median term of $38^{27-42}$ WG with 9 (21\%) before 37 WG and one medical termination of pregnancy for major IUGR at 21WG. IUGR was observed in 6 pregnancies (14\%) associated with hypertension and pre-eclampsia or HELLP syndrome in 2 cases.

The median birth weight was 2940 [610-4310] grams. Five children (12\%) required intensive care units hospitalisation. One premature boy (27 WG) died after 2 days. Treatment during pregnancy included steroids $(n=25 / 43,58 \%)$, azathioprine $(n=9 / 43 ; 21 \%)$ and infliximab $(n=1 / 43 ; 2 \%)$. Pre-eclampsia were less frequent in patients treated with steroids during pregnancy $(p=0.02)$.

The risk of developing arterial hypertension was associated with previous chronic arterial hypertension, and an infra-diaphragmatic vasculitis injury $(\mathrm{p}=0.01$ and $p=0.04)$. Activity of TA was observed in the course of $12 / 43$ pregnancies $(28 \%)$. Conclusions: We observed both a high rate of obstetrical complications and of live birth. A preexisting chronic arterial hypertension, the infra-diaphragmatic location of vasculitis and/or an active disease in the 6 months preceding the pregnancy were associated with an impaired pregnancy outcome. TA disease activity did not seem to be influenced by pregnancy.

Disclosure of Interest: None declared

DOI: 10.1136/annrheumdis-2018-eular.4990

\section{FRI0509 THE FACTORS ASSOCIATED WITH ANXIETY/ DEPRESSIVE DISORDERS IN BEHÇET'S DISEASE PATIENTS}

P. Ovcharov $^{1}$, T. Lisitsyna ${ }^{1}$, D. Veltishchev ${ }^{2}$, O. Seravina ${ }^{2}$, O. Kovalevskaya ${ }^{2}$, Z. Alekberova', E. Nasonov'. ${ }^{1}$ Nasonova Research Institute of Rheumatology ${ }^{2}$ Moscow Research Institute of Psychiatry, Moscow, Russian Federation

Background: The anxiety/depressive disorders (ADD) is a special psychopathological problem for Behçet's Disease (BD) patients. ADD has high rates in $B D$, but their causes aren't enough investigated.

Objectives: To determine the main factors associated with anxiety/depressive disorders in BD patients.

Methods: The investigation has been realised in accordance with the interdisciplinary program "Stress factors and mental disorders in immune-mediated inflammatory rheumatic diseases".

$116 \mathrm{BD}$ patients were enrolled in the study. The majority of patients were men $(69,8 \%)$, natives of the North Caucasus $(51,9 \%)$, with mean age $(M \pm m) 33,3 \pm 0,98$ years. All the patients met the criteria of the International Study Group for BD (1990) classification. The disease activity was assessed by scoring system BDCAF.

ADD were diagnosed by psychiatrist in accordance with the ICD-10 in semi-structured interview. The severity of depression was evaluated by MontgomeryAsberg Depression Rating Scale (MADRS) and anxiety - by Hamilton Anxiety Rating Scale (HAM-A). The severity of stress was evaluated by PSS-10 scale. Results: ADD were diagnosed in 87 BD patients (75\%). The dysthymia $(29,3 \%)$ and recurrent depressive disorder $(21,5 \%)$ prevailed in these patients, generalised anxiety disorder $(6,03 \%)$ and single depressive episode $(7,76 \%)$ were rare. Cognitive disorders of different severity were diagnosed in 87 (75\%) patients. The presence of ADD didn't depend on gender and duration of the disease. The factors associated with ADD were found during Pearson correlations. Then linear regression analysis was done and obtaining prognostic model showed that ADD was associated with: sleep disorders $(\beta=0,401)$, asthenia $(\beta=0,176)$, cognitive disorders $(\beta=0,145)$, chronic stress $(\beta=0,036)$ and stress severity (PSS-10 score) $(\beta=0,115)$, age of eye damage $(\beta=0,135)$, onset of ADD before BD onset $(\beta=0,147)$, pure quality of life (QoL) estimated by visual analogue scale (VAS)
( $\beta=0,163)$, gastrointestinal BD symptoms $(\beta=0,101)$, higher CRP level $(\beta=0,174)$, younger age of patients $(\beta=-0,053)$ and early childhood trauma (before 7 years old) $(\beta=0,15)$ (area under the ROC curve $=0,957$ ) (figure 1).

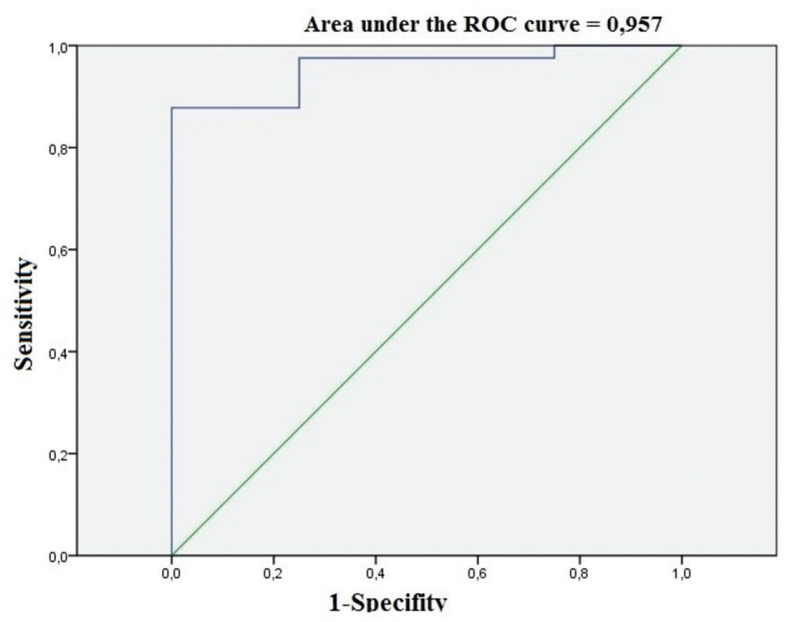

Figure 1 Area under the ROC curve $=0957$

Conclusions: The results demonstrated high prevalence of ADD in surveyed BD patients. $A D D$ in $B D$ is much more associated with early childhood trauma chronic severe stress, onset before BD, younger age of patients, older age of eye damage, gastrointestinal involvement, higher CRP level, accompanied by sleep disturbances, asthenia, cognitive disorders and had a negative impact on QoL.

Disclosure of Interest: None declared

DOI: 10.1136/annrheumdis-2018-eular.6639

\section{FRI0510 INCREASED EXPRESSION OF V-DOMAIN IG SUPPRESSOR OF T-CELL ACTIVATION (VISTA) ON LEUKOCYTES OF GRANULOMATOSIS WITH POLYANGIITIS (GPA) PATIENTS}

R. Hid Cadena ${ }^{1}$, J. Yuan ${ }^{1}$, M.G. Huitema ${ }^{2}$, A.M. Boots ${ }^{2}$, C. Stegeman ${ }^{3}$, E. Brouwer ${ }^{2}$, A. Rutgers ${ }^{2}$, P. Heeringa', W.H. Abdulahad ${ }^{2} .{ }^{1}$ Pathology and Medical Biology; ${ }^{2}$ Rheumatology and Clinical Immunology, ${ }^{3}$ Department of Internal Medicine/Division of Nephrology, University Medical Center Groningen, Groningen, Netherlands

Background: Vascular inflammation in GPA is the result from an inflammatory event combined with a highly specific immune response. Antineutrophil cytoplasmic antibodies (ANCA) specific for GPA are directed against neutrophil granule proteins. Neutrophils are known to play an important role in the pathogenesis of GPA. Under normal conditions, activation of immune cells is positively and negatively regulated by stimulatory and inhibitory checkpoint molecules. The right balance between the expression of both molecules is crucial in fine-tuning the immune response and preventing autoimmunity. Recently, VISTA (V-domain Ig suppressor of $T$ cell activation) has been identified as a potent negative regulator of $T$ cell activation.

Objectives: This study aimed to investigate the expression of VISTA on circulat ing leukocytes of GPA-patients and compare it with vasculitis control (VC) patients with Giant Cell Arteritis (GCA) and healthy controls (HC).

Methods: In a cross-sectional study, fresh blood samples were obtained from 43 GPA-patients in remission on immunosuppressive treatment, $24 \mathrm{VC}$ and 34 sex and age-matched HC. The frequency of VISTA expression was determined on Th-cells (CD45RO ${ }^{-}$and $\mathrm{CD}^{2} 5 \mathrm{RO}^{+}$), NK cells, monocyte subsets (classical/non classical/intermediate) and on neutrophils (suppressive/non suppressive subsets) by flow cytometry.

Results: The proportion of VISTA expressing Th-cells was significantly increased in GPA-patients compared with HCs, this increase could be seen in both, the CD45RO- compartment as well as in the CD45RO ${ }^{+}$compartment. NK cells from GPA-patients showed an increase in the proportion of VISTA expressing cells when compared to the VCs and HCs. Among monocyte subsets, a slight decrease in the proportion of VISTA on the Intermediate subset could be seen. Interestingly, on neutrophils a significant increase in the proportion of VISTA ${ }^{+}$cells was seen in GPA-patients in comparison to HCs and VCs. This increase was most pro nounced in the suppressive neutrophil subset.

Conclusions: VISTA expression is increased in both naïve and memory Th cells of GPA patients in remission. Interestingly, neutrophils of GPA patients showed higher levels of VISTA and this was most pronounced in the suppressive neutrophil subset. Whether the increased expression of VISTA has functional consequences needs further investigation. 